* Corresponding author: B Callus, Department of Biochemistry, La Trobe University, Plenty Road, Bundoora, Victoria 3086 Australia.

Tel: + 6139479 1669; Fax: + 6139479 2467;

E-mail: b.callus@latrobe.edu.au

1. Nakano K, Vousden KH. Mol Cell 2001; 7: 683-694.

2. Yu J, Zhang L, Hwang PM, Kinzler KW et al. Mol Cell 2001; 7: 673-682.
3. Villunger A, Michalak EM, Coultas L et al. Science 2003; 302: 1036-1038.

4. Strasser A. Nat Rev Immunol 2005; 5: 189-200.

5. Chipuk JE, Bouchier-Hayes L, Kuwana T et al. Science 2005; 309: 1732-1735.

6. Erlacher M, Michalak EM, Kelly PN et al. Blood 2005; 106: 4131-4138.

7. Jeffers JR, Parganas E, Lee Y et al. Cancer Cell 2003; 4: 321-328.

8. You H, Pellegrini M, Tsuchihara K et al. J Exp Med 2006; 203: 1657-1663.

9. Ekert PG, Jabbour AM, Manoharan A et al. Blood 2006; 108: 1461-1468.

\title{
Response to Callus et al on 'Cytoplasmic p53 is not required for PUMA-induced apoptosis'
}

\author{
Cell Death and Differentiation (2008) 15, 215-216; doi:10.1038/sj.cdd.4402248; published online 19 October 2007
}

\section{Dear Editor,}

Mitochondrial outer membrane permeabilization (MOMP) is a critical event in the mitochondrial pathway of apoptosis, and this is effected and affected by the different members of the BCL-2 family of proteins.

Our paper by Chipuk et al introduced the concept that complex interactions between the different types of BCL-2 proteins extends to interactions with non-BCL-2 family proteins, such as p53. Specifically, we showed that the ability of cytosolic p53 to activate the MOMP effector $\mathrm{BAX}^{2}$ is inhibited by sequestration of cytosolic p53 by $B C L-x L$ (as also suggested by others ${ }^{3}$ ) and, subsequently, de-repressed by displacement of cytosolic p53 by the $\mathrm{BH} 3-$ only protein PUMA. This scenario is an extension of the ideas of Letai and Korsmeyer, ${ }^{4,5}$ upon which we have elaborated. $^{6}$

Callus et al believe that they challenge our conclusions by demonstrating that overexpression of PUMA can cause apoptosis in $p 53^{-1-}$ cells. However, their observation is far from novel, and represents, at best, an incremental extension of the early observations on PUMA we incorporated into our hypothesis. The original descriptions of PUMA demonstrated that its ectopic overexpression triggered apoptosis in the p53null cell line $\mathrm{H}_{1299^{7}}$ and in HCT116 $p 53^{-/-}$. $^{8}$ Studies using cells from puma knockout animals showed roles for PUMA in forms of apoptosis that are known to be p53-independent. ${ }^{9-12}$ In our paper ${ }^{1}$ (Supplementary Figure 4D and E) and in another study, ${ }^{13}$ we showed that the PUMA BH3 domain peptide sensitized cells to apoptosis induced by BID, BIM, or staurosporine, in a p53-independent manner. The model we developed was with full knowledge of these findings, and is misrepresented by Callus et al whose observations do not contradict our results.

Callus et al also imply that our experimental use of HCT116 $p 53^{-1-}$ cells was not properly controlled, as we had not ruled out additional, undefined mutations in the apoptotic pathways of these cells. On the contrary, we demonstrated in Chipuk et al Supplementary Figure 4D that HCT116 wild-type and $p 53^{-1-}$ responded similarly to staurosporine treatment indicating that apoptotic signaling upstream of mitochondria is intact. Furthermore, in Supplementary Figure 4E, HCT116 wild-type, $p 53^{-1-}, p 21^{-1-}$ and $p 21^{-1-}$ Puma $^{-1-}$ cells responded almost identically to the BID and $\mathrm{BIM} \mathrm{BH} 3$ domain peptides, arguing against any major defects in the mitochondrial pathway of apoptosis in the cells we employed.

While ectopic overexpression of PUMA can cause apoptosis in some cell lines, ${ }^{7,8}$ a simple search for the constitutive endogenous expression of Puma in normal human tissues, pathology samples and developmental stages using the NCBI UniGene EST database ${ }^{14}$ clearly indicates that PUMA is not, itself, sufficient to trigger apoptosis in all cases. Furthermore, there are numerous common laboratory cell lines that constitutively express PUMA protein in the absence of proapoptotic treatment (e.g., K562, HT1080, A204, NIH3T3, MOLT4, and U937); and more recently, two reports of constitutive PUMA expression in mouse embryonic fibroblasts. ${ }^{15,16}$ To understand this apparent paradox, it is necessary to consider the role of direct activator versus sensitizer/de-repressor BH3-only proteins in the control of BAX and/or BAK activation (Figure 1), as discussed in more detail elsewhere. ${ }^{6}$ In general, cells that are transformed or otherwise stressed (e.g., by culture conditions) can become 'addicted' to the antiapoptotic members of the BCL-2 family, such that de-repression will release an activator of BAX and/or BAK to trigger MOMP and apoptosis. ${ }^{5}$ Activators include BID, $\mathrm{BIM}$, and cytosolic p53, and probably other proteins. In addition, nonprotein activators of BAX and/or BAK (e.g., heat, $\mathrm{pH}$ extremes) have also been described suggesting that numerous proapoptotic pathways converge on promoting an active multidomain conformation. ${ }^{6,17,18}$ In contrast, PUMA acts as a de-repressor. While we cannot exclude that at some concentrations or under some conditions PUMA may 


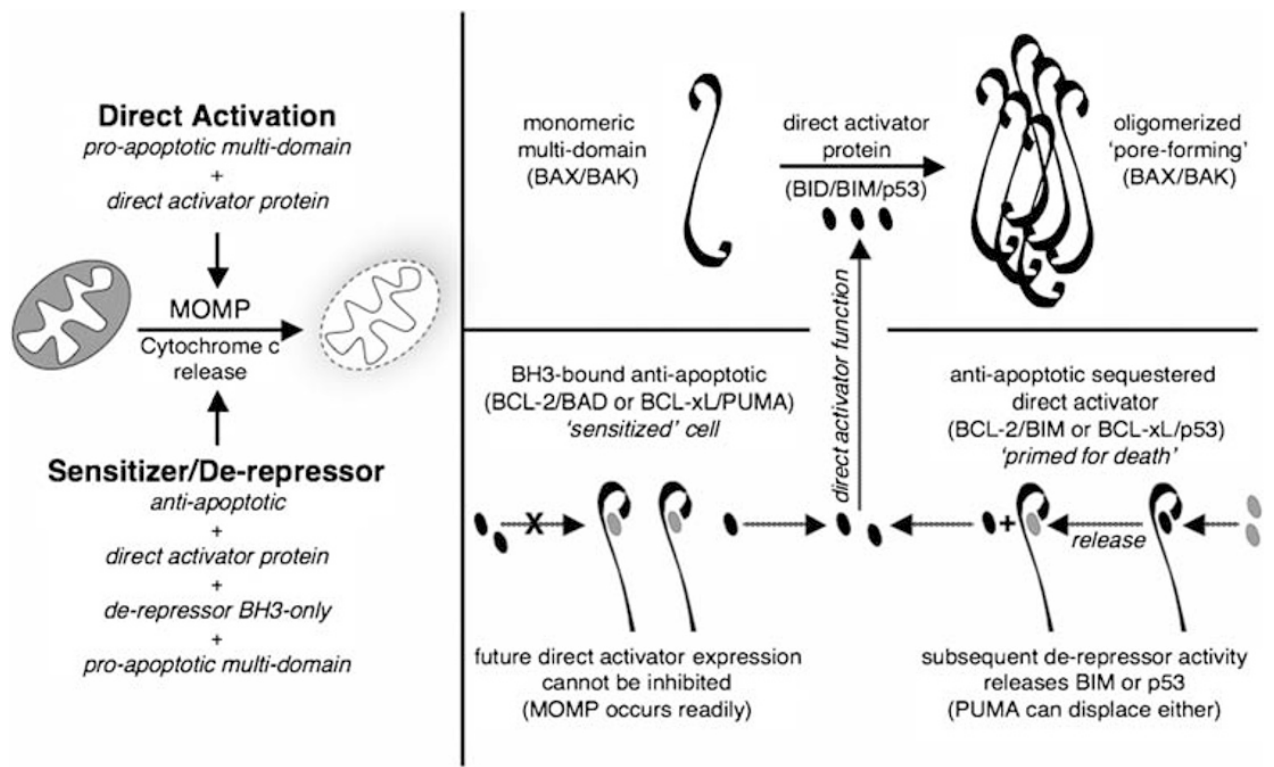

Figure 1 Mitochondrial outer membrane permeabilization (MOMP) requires multiple interactions within the BCL-2 family of proteins. In general, there are two routes to MOMP: (1) a direct activator protein, such as BID, BIM or cytosolic p53 is engaged and interacts with a proapoptotic multidomain (i.e., BAX and/or BAK) to induce its activation and pore forming function, top panel. Non-protein activators of BAX and/or BAK (e.g., heat, pH extremes, and detergents/lipids) may also contribute but are not shown; (2) multiple BCL-2 proteins interact via a sensitization or de-repression network that promotes MOMP because of antiapoptotic proteins sequestering and then releasing BH3-only proteins (e.g., BID or BIM) or other direct activators (e.g., cytosolic p53) in response to collateral BH3-only protein expression, bottom panel. In this $\mathrm{BCL}-2$ family network, PUMA expression can release any direct activator protein to induce MOMP. While PUMA specifically disrupts the p53/BCL-xL interaction, PUMA does not discriminate between other direct activator/antiapoptotic complexes, such as BID/BCL-xL, BID/MCL-1, or BIM/BCL-xL, all of which are potent inducers of MOMP and apoptosis

directly activate BAX and/or BAK to induce MOMP, as suggested, ${ }^{19}$ we have not found evidence for this in our work ${ }^{1}$ (and results not shown). Recently, pharmacologic support for our model was presented in which an inhibitor of cytosolic p53 function effectively blocked DNA damage-induced cell death in vitro and in vivo, without blocking the expression of PUMA. ${ }^{20}$

Thus, our work, along with many others, suggests that PUMA acts by releasing direct activators of BAX and BAK (e.g., BID, BIM, or p53) from antiapoptotic BCL-2 family proteins (such as $B C L-x L$ ) that sequester them. This scenario may be p53 dependent, or not, depending on the cells, conditions of sensitization, and treatment. The argument of Callus et al is specious, as it is an oversimplification and misrepresentation of our conclusions.

\section{JE Chipuk ${ }^{1}$ and DR Green ${ }^{* 1}$}

1 Department of Immunology, St Jude Children's Research Hospital, $332 \mathrm{~N}$. Lauderdale Street, E7050, Memphis, TN 38105-2794, USA
* Corresponding author: DR Green, Department of Immunology, St Jude Children's Research Hospital, 332 N. Lauderdale Street, E7050, Memphis, TN 38105-2794, USA.

Tel: 901495 3470; Fax: 901495 5766; E-mail: douglas.green@stjude.org

1. Chipuk JE, Bouchier-Hayes L, Kuwana T et al. Science 2005; 309: 1732-1735.

2. Chipuk JE, Kuwana T, Bouchier-Hayes L et al. Science 2004; 303: 1010-1014.

3. Mihara M, Erster S, Zaika A et al. Mol Cell 2003; 11: 577-590.

4. Letai A, Bassik MC, Walensky LD et al. Cancer Cell 2002; 2: 183-192.

5. Certo M, Del Gaizo Moore V, Nishino M et al. Cancer Cell 2006; 9: 351-365.

6. Green DR. Cancer Cell 2006; 9: 328-330.

7. Nakano K, Vousden KH. Mol Cell 2001; 7: 683-694.

8. Yu J, Wang Z, Kinzler KW et al. Proc Natl Acad Sci USA 2003; 100: 1931-1936.

9. Jeffers JR, Parganas E, Lee $Y$ et al. Cancer Cell 2003; 4: 321-328.

10. Villunger A, Michalak EM, Coultas L et al. Science 2003; 302: 1036-1038.

11. You H, Pellegrini M, Tsuchihara K et al. J Exp Med 2006; 203: 1657-1663.

12. Erlacher M, Labi V, Manzl C et al. J Exp Med 2006; 203: 2939-2951.

13. Kuwana T, Bouchier-Hayes L, Chipuk JE et al. Mol Cell 2005; 17: 525-535.

14. Expression profile suggested by analysis of EST counts for BBC3/Puma. http://www.ncbi. nlm.nih.gov/UniGene/ESTProfileViewer.cgi?uglist $=\mathrm{Hs} .467020$.

15. Uren RT, Dewson G, Chen L et al. J Cell Biol 2007; 177: 277-287.

16. Willis SN, Fletcher JI, Kaufmann T et al. Science 2007; 315: 856-859.

17. Pagliari LJ, Kuwana T, Bonzon C et al. Proc Natl Acad Sci USA 2005; 102: 17975-17980.

18. Cartron PF, Oliver L, Mayat E et al. FEBS Lett 2004; 578: 41-46.

19. Kim H, Rafiuddin-Shah M, Tu HC et al. Nat Cell Biol 2006; 8: 1348-1358.

20. Strom E, Sathe S, Komarov PG et al. Nat Chem Biol 2006; 2: 474-479.

\section{Supplementary Information accompanies the paper on Cell Death and Differentiation website (http://www.nature.com/cdd)}

